\title{
Differing Host Exploitation Efficiencies in Two Hyperparasitoids: When is a 'Match Made in Heaven'?
}

\author{
Jeffrey A. Harvey • Roel Wagenaar • Rieta Gols
}

Revised: 16 December 2010 / Accepted: 22 December 2010 /

Published online: 15 January 2011

(C) The Author(s) 2011. This article is published with open access at Springerlink.com

\begin{abstract}
Host exploitation behavior in two hyperparasitoids, Lysibia nana and Gelis agilis, was compared in single cocoon clusters of their primary parasitoid host, Cotesia glomerata. L. nana reproduces sexually, is fully winged, does not host-feed and matures eggs quite rapidly after eclosion, whereas G. agilis possesses opposite traits. Cohorts of individual hyperparasitoid females of differing age and physiological state were given access to single cocoon clusters of C. glomerata that also varied in age. These results reveal that the reproductive biology of L. nana is well matched to exploit cocoon broods in $C$. glomerata, suggesting strong a co-evolutionary history with this host. By contrast, G. agilis is much less efficient at exploiting host cocoons and is probably a generalist species that attacks other hosts in nature.
\end{abstract}

Keywords Cotesia glomerata $\cdot$ host exploitation · egg load · Gelis agilis · hostfeeding $\cdot$ host quality $\cdot$ Lysibia nana $\cdot$ oviposition

\section{Introduction}

The evolution of patch- and host-exploitation behavior in parasitoid wasps has been studied for many years (Hassell 1971; Mackauer and Völkl 1993; Spataro and Bernstein 2007). Parasitoids are model organisms for ecological and evolutionary studies because many species can only develop on certain types of hosts which are patchily distributed in nature (Godfray and Shimada 1999). Given this fact, female

\footnotetext{
J. A. Harvey $(\bowtie) \cdot$ R. Wagenaar

Department of Terrestrial Ecology, Netherlands Institute of Ecology, Droevendaalsesteeg 10, 6708 PB

Wageningen, The Netherlands

e-mail: j.harvey@nioo.knaw.nl

R. Gols

Department of Entomology, Wageningen University and Research Centre, P.O. Box 8031, 6700 EH

Wageningen, The Netherlands 
parasitoids are under strong selection to optimize the exploitation of host patches in order to optimize their fitness. One of the shortcomings of studies exploring patch residence rules in parasitoids is that they often pay little attention to a range of ecophysiological factors that influence foraging behavior. For example, reproductive traits often differ profoundly amongst parasitoids attacking different stages of the same host, and even in some instances the same stage (Price 1970, 1972; Harvey 2008; Harvey et al. 2009). Another constraint on parasitoid foraging is based on the number of eggs that the parasitoid can immediately mobilize for oviposition (Jervis et al. 2001). Many parasitoids are synovigenic and emerge with only a few (or even no) ripe eggs and require up to $48 \mathrm{~h}$ or more to accumulate significant numbers (Jervis et al. 2001, 2008). Depending on constraints imposed by host abundance and/ or availability, some parasitoids invest very little in reproduction and thus never produce large complements of eggs (Ellers et al. 2000).

Another important factor influencing the evolution of foraging behavior in parasitoids concerns the degree of host specialization exhibited by the parasitoid species being studied. Although generalists have the advantage of exploiting a wider range of resources, they are predicted to be less efficient in using one particular resource compared to specialists (Stilmant et al. 2008). Highly specialized parasitoids that attack one or only a few hosts in nature will exhibit behavior and biological characteristics that are finely tuned to hosts with which they are strongly co-evolved (Price 1970, 1972; Godfray 1994). By contrast, the foraging behavior of broad generalists will probably be less tailored to a specific host species and therefore their response to host patches may be less refined than that demonstrated by specialists.

In this study we compare and contrast the responses of two secondary idiobiont hyperparasitoids to single broods of their primary parasitoid host, Cotesia glomerata L. (Hymenoptera: Braconidae). C. glomerata is a gregarious primary koinobiont endoparasitoid that attacks the larvae of cabbage butterflies (Pieridae). In turn, cocoons of $C$. glomerata are attacked by a number of secondary hyperparasitoids, including the solitary species Lysibia nana Gravenhorst (Hymenoptera: Ichneumonidae) and Gelis agilis Fabricius (Hymenoptera: Ichneumonidae). Both hyperparasitoids are quite closely related (they occur in the same subfamily, Cryptinae) although L. nana is much more frequently recovered from cocoon clusters of $C$. glomerata (Harvey 2008; Harvey et al. 2009). At eclosion, both species have no mature eggs in their ovaries and thus are wholly synovigenic (Jervis et al. 2008). However, the two species differ in some important respects. For example, L. nana reproduces sexually and adults are fully winged, whereas G. agilis is an asexually reproducing species whose females are wingless. Furthermore, L. nana acquires all of its resources for reproduction during larval development, whereas adult G. agilis must host-feed to optimize egg production (Jervis and Kidd 1986; Harvey 2008). Finally, although the adult wasps are of equivalent size, eggs of G. agilis are much larger than those of L. nana (Harvey 2008).

Cotesia glomerata typically produces single broods of tightly clustered cocoons containing an average of 20-30 wasps per brood (Harvey 2000; Gu et al. 2003). These clusters represent an aggregated and high value resource for solitary hyperparasitoids, and it has been reported that some hyperparasitoid females attend cocoon broods of their gregarious hosts over several days, parasitizing a number each day (based on the rate of egg maturation) and guarding against con- or 
heterospecfic females (Takai et al. 2008). The aim of the current experiment is to determine how effectively individual females of $L$. nana and $G$. agilis exploit clusters of $C$. glomerata containing 25 cocoons when they are confined to a single cluster throughout their lives. Given that the quality of $C$. glomerata pupae declines rapidly with age (Harvey et al. 2006), the response of female hyperparasitoids to different age cohorts of $C$. glomerata cocoons was also measured. Lastly, the physiological state of the two hyperparasitoids was also manipulated (adult female age in both species, host-feeding experience in G. agilis) in order to determine if this affects their oviposition behavior. Our main hypothesis is that L. nana, which is more specialized on $C$. glomerata, will numerically and temporally exploit cocoon clusters much more effectively than G. agilis, which is more of a generalist.

\section{Methods and Materials}

Insects

Hosts and parasitoids were maintained at $25 \pm 2{ }^{\circ} \mathrm{C}$ under a $16: 8 \mathrm{~h} \mathrm{~L}: \mathrm{D}$ regime. Cultures of $C$. glomerata and P. brassicae were obtained from insects reared at Wageningen University (WUR), The Netherlands. These had been originally collected from agricultural fields in the vicinity of the University. All P. brassicae larvae used in these experiments had been maintained on Brassica oleracea cv. Cyrus (Brussels sprouts) at WUR.

Cotesia glomerata were reared according to the protocol described in Harvey (2000). Adult female wasps typically oviposit 10-40 eggs into first (L1) to third (L3) instars of $P$. brassicae. During their development the parasitoid larvae feed primarily on host hemolymph and fat body. When they are mature, the larvae emerge from the host caterpillar late during its final instar, and they immediately spin cocoons on the host plant adjacent to the host, which perishes within a few days.

Lysibia nana was originally obtained from cocoons of $C$. glomerata recovered from leaves of $B$. nigra growing in a garden plot adjacent to the Institute of Ecology, Heteren, The Netherlands. It is a fairly specialized species and thus attacks several closely related gregarious endoparasitoid species in the higher Microgastrinae that pupate in exposed locations on the primary host foodplant. $C$. glomerata appears to be one of its preferred hosts in nature (Harvey 2008). Adult females of L. nana species perforate the host cocoon with their ovipositor and inject permanently paralysing venom into the pre-pupa or pupa. Following envenomation, the wasps lay a single egg on the moribund host. After the parasitoid egg hatches, the larva chews a hole through the host cuticle with its mandibles and imbibes hemolymph, but as it grows it begins attacking other tissues indiscriminately and ultimately consumes the entire host within a few days, pupating within the cocoon produced by $C$. glomerata. Female wasps emerge with no mature eggs but quickly mobilize stored resources and mature a full egg load within 4 days of eclosion (Harvey 2008; Harvey et al. 2009).

Gelis agilis was also originally obtained from cocoons of C. glomerata placed into the field adjacent to the Institute. This species differs from L. nana in that egg production is contingent on host-feeding activity by the adult female parasitoid. 
Proteins obtained from the hemolymph are utilized by the parasitoid for egg production (Harvey 2008). Once mature eggs are available, oviposition behavior in G. agilis is similar to that in L. nana. In culture, L. nana and G. agilis were maintained exclusively on 1-2 day-old pupae of $C$. glomerata. After emergence, hyperparasitoids were kept in large $\left(20 \mathrm{~cm}\right.$ dia.) Petri dishes at $10^{\circ} \mathrm{C}$.

\section{Experimental Protocol}

Larvae of $P$. brassicae were initially parasitized by females of $C$. glomerata in the first instar (L1) in rearing cages $(35 \times 35 \times 35 \mathrm{~cm})$. Cabbage leaves containing $>300$ L1 larvae were placed into cages containing male and female parasitoids. The leaves were attached to the side of the cage by inserting ordinary pins through the stem and the cage mesh; this allows easier access of the wasp to the caterpillars. After $30 \mathrm{~min}$ the leaves were removed from the parasitoid cages and the larvae were transferred to undamaged cabbage plants in larger rearing cages $(1 \mathrm{~m} \times 60 \mathrm{~cm} \times 60 \mathrm{~cm})$. Each cage contained four food plants that were refreshed every 3 days, or earlier if required. When the parasitoid larvae egressed from the host caterpillar, they were allowed to spin cocoons and form individual clusters. Because parasitoid cocoon clusters are fragile for several hours after being constructed by the parasitoid larvae, they were left in the cages for several hours. After this time (which was recorded), the cocoon clusters were gently collected from plants or the cage walls and were taken back to the laboratory where they were carefully separated using a pair of forceps and tweezers. Individual cocoons of $C$. glomerata were then transferred to large Petri dishes (20 cm dia.) according to specific age cohorts based on their day of emergence.

In order to determine the mean size of individual broods of $C$. glomerata in the field, individual L1 larvae were singly parasitized by female $C$. glomerata wasps and allowed to develop on cabbage plants (as described above). Ten plants were placed outside in late summer for 1 week; each plant initially contained approximately 25 larvae. After they were collected the larvae were reared as described above. From these larvae the mean brood size of $C$. glomerata was $24.38( \pm 1.26)$. This, in combination with the results from other studies (e.g. Harvey 2000; Tagawa 2000) enabled us to assume that a brood size of 25 well approximates that produced by C. glomerata.

Twenty five cocoons were then counted from one of 4 age cohorts: 12 h-old, 36 h-old, 60 h-old or 84 h-old. These ages were selected on the basis of host quality for the hyperparasitoids which has been shown for $L$. nana to decline with time (Harvey et al. 2006). Individual females of L. nana or G. agilis were placed individually into Petri dishes (12 cm dia) containing 25 cocoons from a specific age cohort (thus, one hyperparasitoid per dish). Honey and water were also constantly provided. Individual wasps were given consistent access to the same 25 cocoons for the following 7 days (which is significantly longer than the time required for C. glomerata to complete its development and emerge as adult wasps) and were then removed from the Petri dishes. The main reason for allowing the hyperparasitoids extended access to single broods is to see how effectively these broods are exploited and if this co-varies with the age of the cocoons and the age and physiological state of the hyperparasitoid. Therefore, the fitness of individual females was partly 
dependent on the fraction of the 25 cocoons that they parasitized and in which their progeny successfully eclosed as adults. The fate of the cocoons was then monitored as either the emergence of adult L. nana or G. agilis or the emergence of adult $C$. glomerata. Cocoons that failed to produce hyperparasitoids or primary parasitoids were dissected after 2 months and their fate was determined as the following: dead fully developed L. nana, G. agilis or C. glomerata pupae, or unknown (e.g. a dead parasitoid larva). Furthermore, in order to determine if the physiological state of the hyperparasitoid affects their response to cocoons of different age, certain traits of both species were manipulated. In L. nana, newly eclosed wasps as well as 96 h-old (= 4 days) wasps were provided with cocoons of $C$. glomerata. The same criteria were used with $G$. agilis, with the exception of an additional treatment: 96 h-old wasps that had been given access to cocoons of $C$. glomerata (for host-feeding purposes) for $48 \mathrm{~h}$ prior to the experiment. Each combination of host-age and parasitoid state was replicated 10 times. Thus a total of 80 females of L. nana and 120 females of $G$. agilis were used.

An earlier study (Harvey 2008) found that the eggs of G. agilis were larger than eggs of L. nana in adult female wasps of comparable size. In order to determine if the time required to lay an egg (= oviposition time) in experienced wasps differed in the two species, $20 \mathrm{~L}$. nana and $G$. agilis females that were at least 5 days old were given access to cocoons of $C$. glomerata for 2 days. After this time the cocoons were removed for an additional 2 days. The same wasps were then presented with individual cocoons of $C$. glomerata in small Petri dishes $(5.5 \mathrm{~cm} \mathrm{dia.)}$ and their oviposition times were recorded. This was based on the number of seconds between ovipositor insertion and removal from host cocoons.

\section{Statistical Analyses}

A generalized linear model with a binomial distribution for errors and a logit link function was used to test whether the fate of $C$. glomerata cocoons that were hyperparasitized by either L. nana or G. agilis differed depending on the physiological state of the hyperparasitoid and the age of the $C$. glomerata cocoons. The physiological state (newly eclosed, 96 h-old, or host fed only in G. agilis), the age of the $C$. glomerata cocoons (12, 36, 60 or 84 h-old), as well as their interaction term were entered as explanatory variables in the regression model. The response variables were the number of $C$. glomerata cocoons out of $25(n=10)$ that produced (1) live hyperparasitoids, (2) dead hyperparasitoids, or (3) live C. glomerata, or (4) dead $C$. glomerata. Cocoons that did not produce a live parasitoid, but for which it could not be determined whether they had developed into a primary or secondary parasitoid were categorized as (5) unknown death. Each of the five response variables was analyzed separately.

\section{Results}

There were significant effects of treatment (= hyperparasitoid age), host cocoon age and the interaction between these parameters for the number of adult L. nana emerging, $C$. glomerata emerging and the number of unidentified dead larvae found 
in dissected cocoons (Table 1). The numbers of dead L. nana and C. glomerata did not vary significantly with treatment but did so with cocoon age with a significant interactive effect also observed (Table 1). The results show that, irrespective of female (hyperparasitoid) age, L. nana females successfully parasitized approximately $80 \%$ of cocoons up to 60 h-old (Fig. 1a, b). In the oldest host age class $(84 \mathrm{~h}$ ), successful parasitism declined and was higher in 4 d-old wasps. Survival of C. glomerata, by contrast, was exceptionally low except in the oldest hosts.

All of the observed values in host cocoons parasitized by G. agilis were found to vary significantly with treatment, and all but the number of dead G. agilis that failed to emerge differed significantly with host cocoon age (Table 1). Moreover, there were significant interactive effects of treatment and host age on the number of

Table 1 Logistic regression analysis on the fate of Cotesia glomerata cocoons of different ages (12, 36, 60 or 84 h-old) that were hyperparasitized by either Lysibia nana or Gelis agilis. In L. nana, newly eclosed wasps as well as 96 h-old wasps were provided with $C$. glomerata cocoons. The same criteria were used with $G$. agilis, with the exception of an additional treatment: 96 h-old wasps that had been given access to cocoons of $C$. glomerata (for host-feeding purposes) for $48 \mathrm{~h}$ prior to the experiment. The fate of each cocoon out of 25 per hyperparasitoid physiological status/cocoon-age treatment was recorded either as: (1) live hyperparsitoid; (2) dead hyperparsitoid; (3) live C. glomerata, (4) dead C. glomerata, (5) unknown death

\begin{tabular}{|c|c|c|c|c|c|c|}
\hline \multirow[b]{2}{*}{ Response variable } & \multicolumn{2}{|c|}{$\begin{array}{l}\text { Effect of hyper-parasitoid } \\
\text { treatment (1) }\end{array}$} & \multicolumn{2}{|c|}{ Effect of cocoon age (2) } & \multicolumn{2}{|c|}{$\begin{array}{l}\text { Effect of interaction }(1) \times \\
(2)\end{array}$} \\
\hline & $\begin{array}{l}\text { Deviance ratio (d. } \\
\mathrm{f} .=1)\end{array}$ & $\begin{array}{l}P- \\
\text { value }\end{array}$ & $\begin{array}{l}\text { Deviance ratio }(\mathrm{d} . \\
\mathrm{f} .=3)\end{array}$ & $\begin{array}{l}P- \\
\text { value }\end{array}$ & $\begin{array}{l}\text { Deviance ratio }(\mathrm{d} . \\
\mathrm{f} .=3)\end{array}$ & $\begin{array}{l}P- \\
\text { value }\end{array}$ \\
\hline \multicolumn{7}{|l|}{ Lysibia nana: } \\
\hline $\begin{array}{l}\text { Number of live } \\
\text { Lysibia }\end{array}$ & 40.9 & $<0.001$ & 125 & $<0.001$ & 15.5 & $<0.001$ \\
\hline $\begin{array}{l}\text { Number of dead } \\
\text { Lysibia }\end{array}$ & 1.15 & 0.28 & 7.30 & $<0.001$ & 5.39 & 0.001 \\
\hline $\begin{array}{l}\text { Number of live } \\
\text { Cotesia }\end{array}$ & 30.0 & $<0.001$ & 150 & $<0.001$ & 2.04 & $<0.001$ \\
\hline $\begin{array}{l}\text { Number of dead } \\
\text { Cotesia }\end{array}$ & 1.40 & 0.24 & 72.9 & $<0.001$ & 2.26 & 0.08 \\
\hline Unknown & 9.60 & 0.002 & 30.2 & $<0.001$ & 8.84 & $<0.001$ \\
\hline \multicolumn{7}{|l|}{ Gelis agilis: } \\
\hline $\begin{array}{l}\text { Number of live } \\
\text { Gelis }\end{array}$ & $35.9^{\mathrm{a}}$ & $<0.001$ & 33.3 & $<0.001$ & $0.87^{\mathrm{b}}$ & 0.52 \\
\hline $\begin{array}{l}\text { Number of dead } \\
\text { Gelis }\end{array}$ & $9.85^{\mathrm{a}}$ & $<0.001$ & 1.75 & 0.15 & $1.10^{\mathrm{b}}$ & 0.36 \\
\hline $\begin{array}{l}\text { Number of live } \\
\text { Cotesia }\end{array}$ & $95.9^{\mathrm{a}}$ & $<0.001$ & 117 & $<0.001$ & $7.89^{\mathrm{b}}$ & $<0.001$ \\
\hline $\begin{array}{l}\text { Number of dead } \\
\text { Cotesia }\end{array}$ & $9.50^{\mathrm{a}}$ & $<0.001$ & 4.47 & 0.004 & $9.13^{\mathrm{b}}$ & $<0.001$ \\
\hline Unknown & $22.5^{\mathrm{a}}$ & $<0.001$ & 112 & $<0.001$ & $2.29^{\mathrm{b}}$ & 0.03 \\
\hline
\end{tabular}




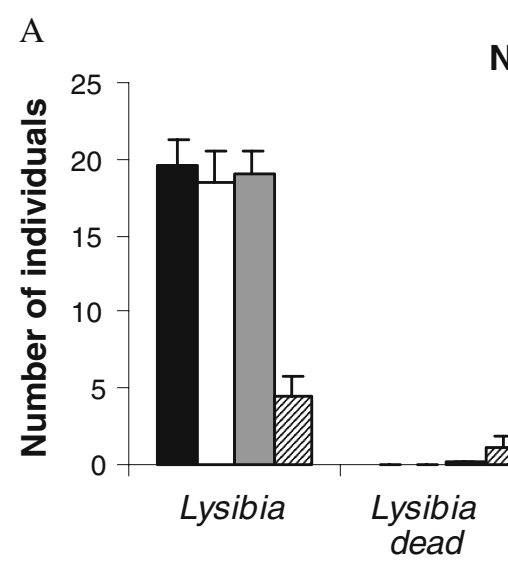

Newly emerged

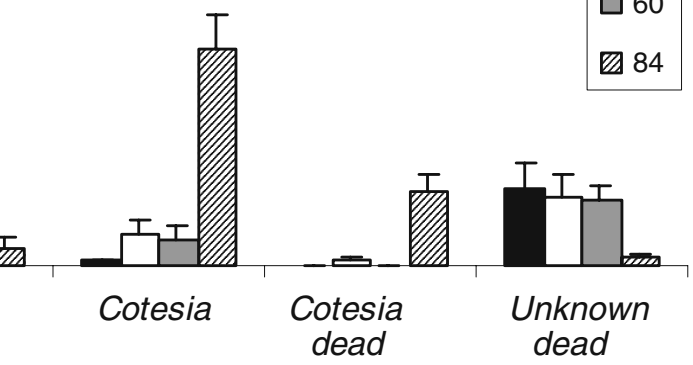

Fate

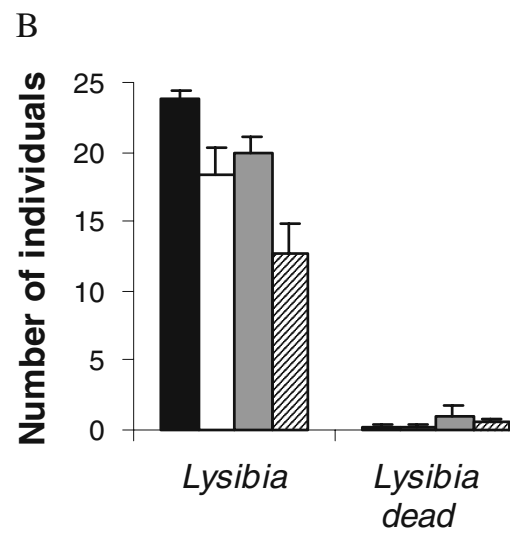

4 days old

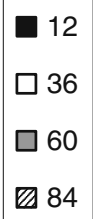

84

Fig. 1 Fate of Cotesia glomerata cocoons of different presented to Lysibia nana females that were a newly emerged, or b 4 days old. Host cocoons were either 12, 36, 60 or 84 h old when presented to female hyperparasitoids. Line bars represent standard error of the mean. Each treatment was replicated 10 times

C. glomerata surviving to eclosion, as well as on the number of dead primary parasitoids and unknown larvae recovered from dissected cocoons (Table 1). The results with $G$. agilis markedly differed from those shown by L. nana. Irrespective of host cocoon age and hyperparasitoid treatment, G. agilis females were only able to successfully exploit a small fraction of available hosts. Moreover, this declined with host age (Fig. 2a-c). Hyperparasitoids that had been given access to hosts for hostfeeding purposes also produced more progeny in hosts of a given age than naïve hyperparasitoids.

\section{Discussion}

The number of cocoons (25) provided to female wasps of both hyperparasitoid species in this study closely approximates brood sizes of $C$. glomerata under both 
natural and laboratory conditions (this study; Harvey 2000; Tagawa 2000; Gu et al. 2003). We found that L. nana was much more efficient in parasitizing individual cocoon clusters of $C$. glomerata than its close relative, G. agilis. Moreover, this efficiency was independent of the age of female wasps used in the experiment. In hosts aged up to $60 \mathrm{~h}$, L. nana wasps emerged from approximately $80 \%$ of cocoons, with the concomitant survival of $C$. glomerata being very low. In some replicates, every cocoon was successfully parasitized by L. nana. Only in 84-h old hosts did L. nana emergence decline sharply, and even here older wasps produced more progeny than wasps in any of the G. agilis-host age treatments. By contrast, $G$. agilis emerged from a small fraction of available host cocoons, and this uniformly declined with host age. It also appeared that $G$. agilis killed many $C$. glomerata pupae by destructively host-feeding on them, a behavior that is important in that it enables the hyperparasitoid females to accrue the proteins necessary to produce eggs. However, even when wasps had been given previous access to cocoons in which they could host-feed, the most offspring produced by G. agilis averaged 7 (in 12-h old cocoons).

These results reveal that the reproductive biology of L. nana is strongly tailored to individual broods of $C$. glomerata whereas in $G$ agilis it is not. Although both species emerge with no eggs, L. nana mobilizes resources carried over from larval feeding and the female wasp's ovaries accrue eggs rapidly over the following 2-3 days. This enabled $L$. nana to exploit most cocoons within a single cluster, even when they were older. Maximum egg loads of L. nana females reach about 40 within several days of eclosion, which represents the upper threshold of single $C$. glomerata broods in nature. Individual L. nana females that are able to locate individual cocoon clusters may effectively 'hit the jackpot' and experience significant fitness returns because they are able to exploit a large proportion of the cluster.

On the other hand, on cocoon clusters of $C$. glomerata, female G. agilis wasps experienced both time and egg limitation and were thus only able to exploit a small proportion of host cocoons for host-feeding and reproductive purposes. Resources carried over from larval feeding are insufficient for G. agilis to produce more than 1 or 2 eggs, and thereafter the wasp must host-feed to obtain further resources for oögenesis. Even then egg loads in female wasps never exceed 3, and these eggs are of much greater volume than eggs of L. nana. As a result, even when provided with unlimited cocoons of $C$. glomerata, the most progeny that G. agilis can produce over the course of $24 \mathrm{~h}$ is three or four (Harvey 2008). Furthermore, G. agilis is wingless and its spatial area of search is much smaller than that of L. nana. In evolutionary terms, species of Gelis have overcome this handicap by becoming broad generalists. In addition to a wide range of parasitoid cocoons, various species in this genus are known to attack and successfully parasitize such phylogenetically divergent hosts as lacewings, moth pupae and spider egg sacs (Bezant 1956; van Baarlen et al. 1996; Cobb and Cobb 2004; Finch 2005).

Given that suitable hosts are patchily distributed and may be scarce it may pay for a female hyperparasitoid to remain in the vicinity of an individual cocoon cluster and to parasitize them at their physiological limit. This is because the lifetime reproductive success of a female wasp may be based on her ability to exploit that single cluster, at least if suitable hosts are hard to find. For example, the solitary hyperparasitoid 


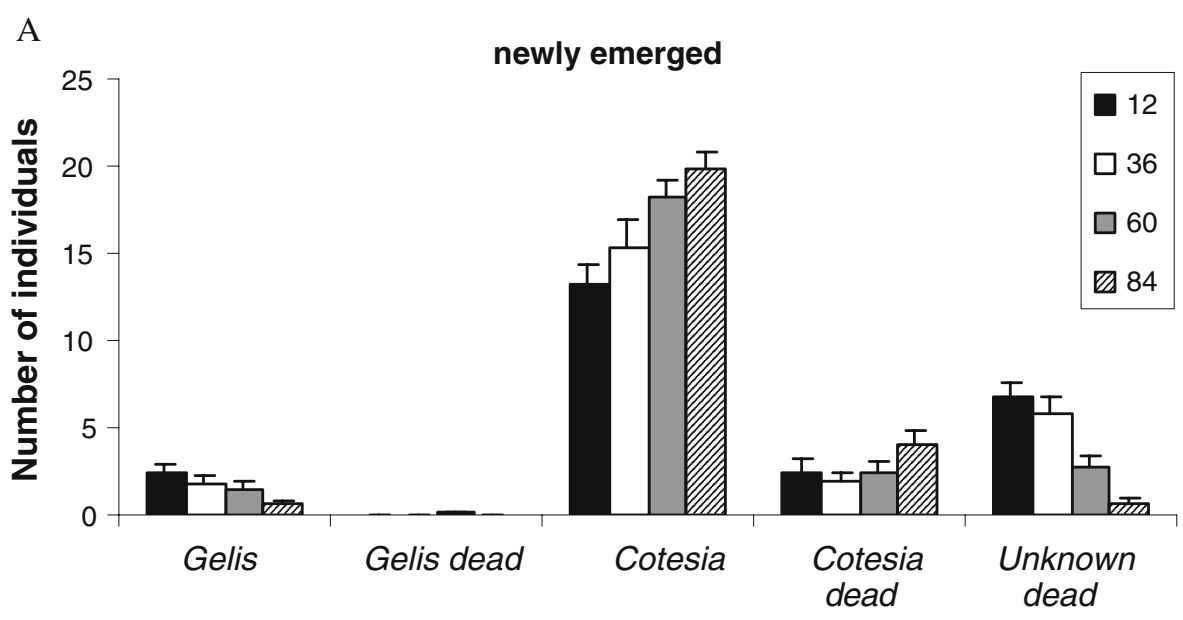

Fate

B

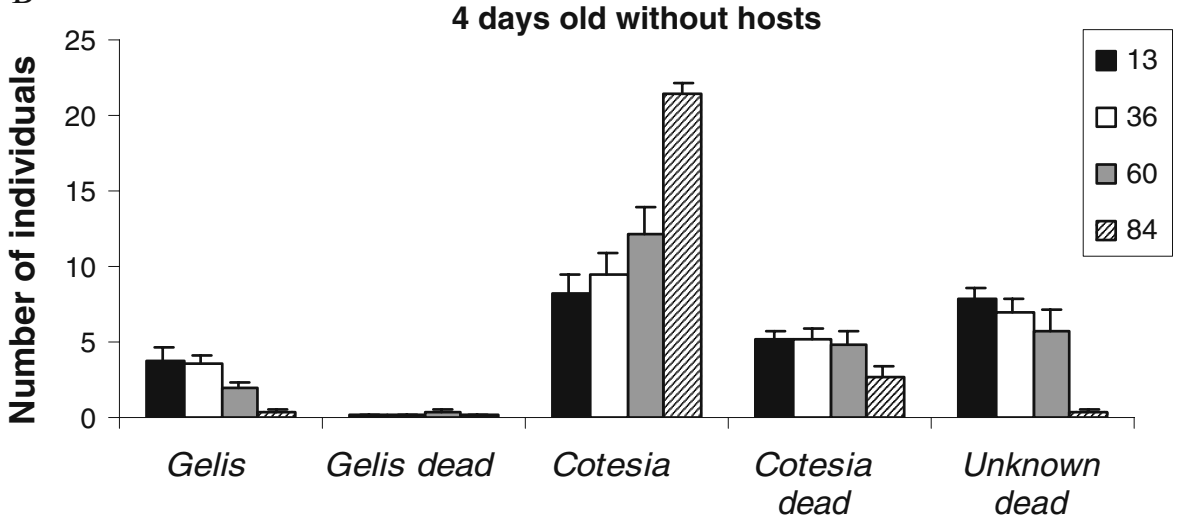

Fate

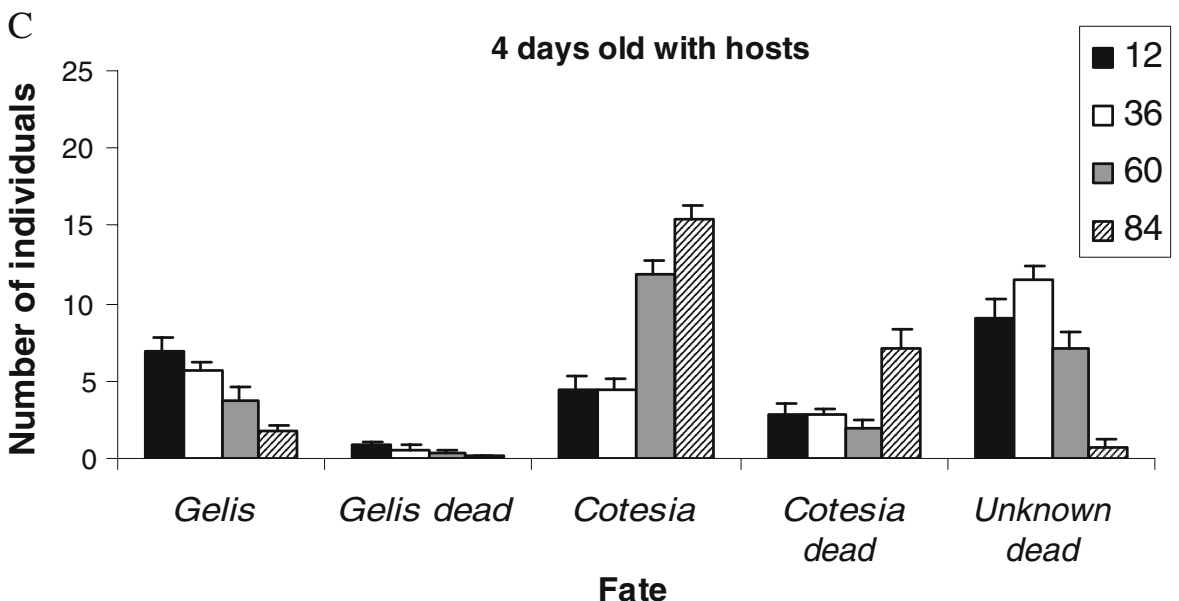


Fig. 2 Fate of Cotesia glomerata cocoons of different ages presented to Gelis agilis females that were a newly emerged, b 4 days old without host access, or c provided with hosts between days 2 and 4 posteclosion. Host cocoons were either 12, 36, 60 or 84 h old when presented to female hyperparasitoids. Line bars represent standard error of the mean. Each treatment was replicated 10 times

Trichomalopsis apanteloctena remains on cocoons clusters of its primary parasitoid host, Cotesia kariyai, for up to $120 \mathrm{~h}$, and parasitizes 10-20 of them per day (Takai et al. 2008). During this time the females will attack any other female hyperparasitoids that approach the cocoons. Several other species of idiobiont parasitoids are also known to aggressively defend cocoon clusters by driving off other (hyper) parasitoid females that attempt to gain access to them (Hardy and Blackburn 1991; Zaviezo and Mills 2000). Like G. agilis, the amount of per capita investment into parasitoid eggs is very high in these species, meaning that eggs represent a very costly investment. Brood guarding appears to have evolved in parasitoids with long handling times and where the risk of host attack from other con- or heterospecific females is high (Takai et al. 2008). Under these conditions, cocoon guarding may also be an adaptive strategy in G. agilis.

In pupal parasitoids, host quality declines rapidly with host age (Wang and Liu 2002; Harvey et al. 2006). This is due to sclerotization of the cuticle and the formation of body structures including the head capsule, wings and appendages that become well defined just prior to adult eclosion. At $25^{\circ} \mathrm{C}$, the pupal development of C. glomerata proceeds rapidly, with differentiation of the pupa into specific body structures noticeable within $50-60 \mathrm{~h}$ of pupation. Adult wasps begin emerging with $100 \mathrm{~h}$ of larval emergence from the host caterpillar. Because the tissues of older hosts are largely inaccessible to hyperparasitoid larvae, adult body size and survival in L. nana and G. agilis declines markedly with time. The results here report that the 'window of susceptibility' of C. glomerata pre-pupae and pupae to attack from secondary parasitoids is therefore quite small.

In summary, host exploitation patterns were found to differ profoundly amongst two secondary hyperparasitoids attacking cocoons of the same primary parasitoid host. The response of L. nana to cocoons of its host, C. glomerata that differed in age was much more efficient than that demonstrated in $G$. agilis. These results reveal that reproductive traits in L. nana are well-matched with cocoon broods of $C$. glomerata, whereas in G. agilis they are not, suggesting that the latter is more of an opportunist species. Models of patch time allocation would therefore be expected to differ profoundly between the two species due to differences in their reproductive biology. Future studies exploring host patch exploitation by parasitoids should bear in mind important physiological constraints and in particular the dynamics of egg maturation that may explain observed patterns.

Acknowledgments The authors wish to thank Gregor Disveld for providing experimental facilities and Leo Koopman and André Gidding for constantly supplying larvae of Pieris brassicae and cocoons of Cotesia glomerata. Tibor Bukovinszky from 'Bugs in the Picture' (http://www.bugsinthepicture.com/) kindly supplied the photographs.

Open Access This article is distributed under the terms of the Creative Commons Attribution Noncommercial License which permits any noncommercial use, distribution, and reproduction in any medium, provided the original author(s) and source are credited. 


\section{References}

Bezant ET (1956) Gelis micrurus Forster (Hymenoptera: Ichneumonidae) parasitising a lycosid spider egg sac. Entomol Month Mag 92:106

Cobb LM, Cobb VA (2004) Occurrence of parasitold wasps, Baeus sp and Gelis sp., in the egg sacs of the wolf spiders Pardosa moesta and Pardosa sternalis (Araneae, Lycosidae) in southeastern Idaho. Can Field-Nat 118:122-123

Ellers J, Sevenster JG, Driessen G (2000) Egg load evolution in parasitoids. Am Nat 156:650-665

Finch OD (2005) The parasitoid complex and parasitoid-induced mortality of spiders (Araneae) in a central European woodland. J Nat Hist 39:2339-2354

Godfray HCJ (1994) Parasitoids - behavioral and evolutionary ecology. Princeton University Press, New Jersey

Godfray HCJ, Shimada M (1999) Parasitoids as model organisms for ecologists. Res Popul Ecol 41:3-10

Gu H, Wang Q, Dorn S (2003) Superparasitism in Cotesia glomerata: response of host and consequences for parasitoids. Ecol Entomol 28:422-431

Hardy ICW, Blackburn TM (1991) Brood guarding in a bethylid wasp. Ecol Entomol 16:55-62

Harvey JA (2000) Dynamic effects of parasitism by an endoparasitoid wasp on the development of two host species: implications for host quality and parasitoid fitness. Ecol Entomol 25:267-278

Harvey JA (2008) Comparing and contrasting development and reproductive strategies in the pupal hyperparasitoids Lysibia nana and Gelis agilis (Hymenoptera: Ichneumonidae). Evol Ecol 22:153166

Harvey JA, Vet LEM, Witjes LMA, Bezemer TM (2006) Remarkable similarity in body mass of a secondary hyperparasitoid Lysibia nana and its primary parasitoid host Cotesia glomerata emerging from cocoons of comparable size. Arch Insect Biochem Physiol 61:170-183

Harvey JA, Wagenaar R, Bezemer TM (2009) Life-history traits in closely related secondary parasitoids sharing the same primary parasitoid host: evolutionary opportunities and constraints. Entomol Exp Appl 132:155-164

Hassell MP (1971) Mutual interference between searching insect parasites. J Anim Ecol 40:473-486

Jervis MA, Kidd NAC (1986) Host feeding strategies in hymenopteran parasitoids. Biol Rev 61:395-434

Jervis MA, Heimpel GE, Ferns PN, Harvey JA, Kidd NAC (2001) Life-history strategies in parasitoid wasps: a comparative analysis of “ovigeny.”. J Anim Ecol 70:42-458

Jervis MA, Ellers J, Harvey JA (2008) Resource acquisition, allocation, and utilization in parasitoid reproductive strategies. Ann Rev Entomol 53:361-385

Mackauer M, Völkl W (1993) Regulation of aphid populations by aphidiid wasps: does parasitoid foraging behaviour or hyperparasitism limit impact? Oecologia 94:339-350

Price PW (1970) Characteristics permitting co-existence among parasitoids of a sawfly in Quebec. Ecology 51:445-454

Price PW (1972) Parasitoids utilizing the same host: adaptive nature of differences in size and form. Ecology 53:190-195

Spataro T, Bernstein C (2007) Influence of environmental conditions on patch exploitation strategies of parasitoids. Behav Ecol 18:742-749

Stilmant D, Billinghen C, Hance T, Boivin G (2008) Host specialization in habitat specialists and generalists. Oecologia 156:905-912

Tagawa J (2000) Sex allocation and clutch size in the gregarious larval endoparasitoid wasp, Cotesia glomerata. Entomol Exp Appl 97:193-202

Takai N, Nakamtsu Y, Harvey JA, Miura K, Tanaka T (2008) Brood attending by females of the hyperparasitoid Trichomalopsis apanteloctena (Hymenoptera: Pteromalidae) on cocoon clusters of its host, Cotesia kariyai (Hymenoptera: Braconidae) and its effects on reproduction, development and survival. Eur J Entomol 105:855-862

van Baarlen P, Topping CJ, Sunderland KD (1996) Host location by Gelis festinans, an eggsac parasitoid of the linyphiid spider Erigone atra. Entomol Exp Appl 1:155-163

Wang XG, Liu SS (2002) Effects of host age on the performance of Diadromus collaris (Hymenoptera: Ichneumonidae), a pupal parasitoid of Plutella xylostella (Lepidoptera: Plutellidae). Biocontrol 47:293-307

Zaviezo T, Mills NJ (2000) Factors influencing the evolution of clutch size in a gregarious insect parasitoid. J Anim Ecol 69:1047-1057 